DOI: $10.12737 /$ article_597656dc284855.13131942

УДК 334.7; 65.012.6

АЛГОРИТМ АНАЛИЗА ВЗАИМОДЕЙСТВИЙ КОМПАНИИ С КЛИЕНТАМИ НА РЫНКЕ «BUSINESS TO BUSINESS»

доктор экономических наук, профессор Е. И. Макаров ${ }^{1}$

В. Г. Егоров ${ }^{1}$

1 - Воронежский филиал ФГБОУ ВО «РЭУ им. Г.В. Плеханова», г. Воронеж, Российская Федерация

В настоящее время расширения международных торговых отношений, интенсивно развиваются инструментарии по анализу клиентов. В исследованиях по данной тематике, в большинстве случаев, ставится во главу угла тривиальное правило: «клиент всегда прав». На практике дело обстоит несколько иначе, особенно это касается рынка «business to business». Баланс сил влияния между производителем и клиентом далеко не всегда полностью находится на стороне последнего. Менеджмент компании имеет инструмент по анализу покупателей на основе маркетинговых исследований, но не имеет возможности оценить перспективы и обоюдные выгоды от взаимоотношений с конкретным клиентом. В данном материале приводится алгоритм действий для проведения сортинга, и определения позиции каждого из субъъектов, при ведении переговоров в долгосрочной перспективе. Представленный ниже алгоритм состоит из двух этапов. На первом этапе проводится обзор и дифференцировка покупателей, в зависимости от доходности и перспективности. Конечным результатом первого этапа является сводная таблица, в которой распределены все виды клиентов, в зависимости от их характеристик. Второй этап состоит из отбора наиболее значимых клиентов и анализа внугренних экономических параметров каждого из субъектов, включая компанию-производителя. Далее, составляются неравенства, из которых определяется баланс сил влияния. Использование данного инструмента предоставляет оценку распределения власти между субъектами. Ценность полученной информации выражается в улучшении качества менеджмента в отношении клиентов, а также для дальнейшего использования, в рамках разработки стратегии компании в отношении других экономических агентов.

Ключевые слова: анализ клиентов, виды покупателей, стратегическое планирование, взаимоотношения экономических субъектов

\title{
ALGORITHM OF ANALYSIS OF INTERACTIONS WITH CLIENTS IN THE MARKET «BUSINESS TO BUSINESS»
}

DSc in Economics, Professor E. I. Makarov ${ }^{1}$

V. G. Egorov ${ }^{1}$

1 - Voronezh branch of Federal State Budget Educational Institution of Higher Education «Plekhanov Russian University of Economics», Voronezh, Russian Federation

\begin{abstract}
Currently, the expansion of international trade relations is intensively developing tools for customer analysis. In studies on the subject, in most cases, the trivial rule: "the customer is always right" is at the forefront. In practice, the situation is somewhat different, especially for the market "business to business". The balance of power between the producer and the client not always fully located on the side of the latter one. The company's management has a tool for the analysis of customers based on marketing research, but is not able to assess the prospects and mutual benefits from the relationship with a specific client. In this material the algorithm of actions for carrying out of sorting and determining the position of each of the entities in negotiations in the long term is given. Below algorithm consists of two stages. The first stage involves a review and differentiation of customers, depending on profitability and prospects. The end result of the first stage is a summary table in which all kinds of customers are distributed, depending on their characteristics. The second stage consists of selecting the most important customers and analysis of the domestic economic parameters of each of the entities, including the manufacturer. Next, we compile the inequalities that determine the balance of power of influence. The use of this tool provides an estimate of the distribution of power between the actors. The value of the information received is reflected in the improvement of the quality of management in relation to customers, as well as for future use, in the framework of the development strategy of the company in respect of other economic agents.
\end{abstract}

Keywords: analysis of customers, types of customers, strategic planning, relations of economic actors 


\section{Менеджмент. Экономика. Организация}

Анализу внешнего окружения в частности покупателям и потребителям в настоящее время уделяется достаточно внимания. В частности, это связано с ростом конкуренции на международных и внугренних рынках, как следствие развития маркетинговых технологий. Многие авторы уделяют внимание этой группе внешних экономических агентов $[1,2,3,4]$, а Макаров Е.И., Морковина С.С. также совместно используют различные методы по отбору, анализу и интеллектуальной оценки экономических субъектов $[5,6,7,8]$. Тем не менее практически не исследуется уровень влияния в долгосрочной перспективе между компанией-производителем и клиентами.

Эксперт маркетинга и менеджмента Филип Котлер оценивает сегмент рынка как непривлекательный, если на нем велика рыночная власть покупателей. Угрозу власти он видит в следующих условиях: «Рыночная власть покупателей возрастает в тех случаях, когда: число их не велико или они хорошо организованы; затраты на приобретение продукта составляют значительную часть расходов потребителей; товар однороден; цена переключения на другой продукт незначительна; чувствительность покупателей к ценам, вызванная их низкими доходами высока; существует возможность интеграции покупателей «вверх по течению»» [9, с. 269]. Майкл Портер дополняет этот перечень условий: «Значительный объем продаж, приходящийся на долю покупателя, повышает значимость его бизнеса для продавца. Крупные покупатели представляют особенно мощную силу, если отрасли свойственны высокие постоянные издержки, заставляющие стремиться к полной загрузке мощностей, как это имеет место, например, в переработке зерна или производстве жидких химикатов» [10, с. 62]. Описанные факторы говорят об уверенной позиции покупателя, по отношению к производителю. В свою очередь компания, по таким параметрам как объем продаж, доля выручки, процент чистой прибыли от реализованной продукции, может выделять наиболее влиятельных потребителей. Майкл Портер оставляет власть за клиентом в случае если: «Закупаемая отраслевая продукция составляет значительную долю закупок или издержек покупателя. В этом случае покупатели склонны проводить при закупке тщательный отбор и тратить ресурсы на поиск товаров по благоприятным ценам» $[10$, с. 62]. Теоретики А.А. Томпсон и А.Дж. Стрикленд солидарны с этим мнением, они пишуг: «Чем крупнее потребитель и чем больше покупаемая им доля продукции, тем сильнее его влияние на продавцов» [11, с. 110]. В целом приведенные условия влияния покупателей на компаниюпроизводителя в работах вышеназванных авторов достаточно справедливы. Остановимся на двух последних цитатах М. Портера, а также А.А. Томпсона и А.Дж. Стрикленда. Авторы угверждают, что власть потребителя сильна, если его затраты, приходящиеся на продукт производителя, высоки. Если пойти дальше в рамах данного тезиса, то очевидно, что большее влияние будет оказывать не величина покупателя или объем его закупок, а величина прибыли, которую получает производитель. Высокие затраты, как таковые, не дают преимущества покупателю, особенно без большого числа предложений. Как правило, высокие издержки наблюдаются на те группы товаров/услуг, которые действительно необходимы ${ }^{1}$, в противном случае их следует минимизировать. Раз продукт важен, важен и его продавец. Справедливо сказать, что чем выше спрос, тем больше власть у предложения, и наоборот. Часто за этим тянугся и другие зависимости от производителя, включая сложность или невозможность переключения на другой продукт, послепродажное обслуживание, техническая поддержка и прочие. Приобретение оборудования предприятием или автомобиля физическим лицом, делает их более зависимыми от производителя (гарантийное и техническое обслуживание, запчасти, ремонт и т. д.). В каком-то смысле продукт имеет власть над потребителем, а он, в свою очередь, имеет власть над выбором его поставщика. После того, как выбор сделан, власть потребителя снижается, власть поставщика увеличивается. Эта пропорция и есть одна из целей анализа покупателей и потребителей.

Дэвид Хасси отмечает фактор опытности клиента: «Покупатели, которые имеют дело со сформировавшейся отраслью, обычно более опытны, чем те, которые покупают продукт новой отрасли. Таким образом, чем отрасль более зрелая, тем слабее могуг стать ее позиции в переговорах (конечно, при определенных допущениях)» [12]. Доводы в пользу этого фактора вполне обоснованы, более того, уместно сказать, что чем больше потребитель информирован о производст-

\footnotetext{
${ }^{1} \mathrm{~B}$ частности, для рынка business to business
} 
ве и себестоимости покупаемой продукции, тем более уязвимым становится продавец. Также, важным критерием является наличие запаса времени. Клиент, обладающий ресурсом времени, безусловно, оказывается в более выгодной позиции, чем компанияпроизводитель.

На основании обзора литературы, а также собственных изысканий, можно выделить следующие условия, способствующие усилению власти клиента:

- число покупателей ограничено;

- значительный объем продукции приходится на одного клиента ${ }^{2}$;

- в сегменте высокая конкуренция производителей;

- большое количество товаров заменителей;

- низкая дифференциация продукта;

- высока возможность обратной интеграции;

- осведомленность клиента о технологии и процессе производства;

- наличие у клиента временных ресурсов.

У разных организаций число покупателей может сильно варьироваться. Одни компании имеют широкий список клиентов, другие ограничиваются несколькими крупными покупателями, в частности такое не редко наблюдается на рынке "business to business". Поэтому, целесообразно сформировать перечень основных клиентов и, вспоминая закон Парето, выделить из них наиболее важных. Основными критериями отбора выступают: процент выручки/прибыли, приходящийся на конкретного клиента, объем и частота закупок. Далее, необходимо проанализировать взаимодействия между основными клиентами и компанией, с точки зрения позиции потребителя и его влияния на организацию.

Анализ покупателей разделен на два этапа:

I. Обзор и дифференцирование покупателей, в зависимости от доходности.

II. Определение уровня влияния между фирмой и клиентами.

Первый этап заключается в формировании списка клиентов, для дальнейшего сортинга по группам.

Ниже изложен ряд действий в рамках І-го этапа:

1. Сбор информации о прибыли компании,

2 Чем большую прибыль приносит компании клиент, тем в большей степени сильнее его влияние от реализуемой продукции, приходящейся на долю клиентов.

2. Составление списка клиентов.

3. Ранжирование клиентов по количеству прибыли, приходящейся от каждого (в процентном соотношении к общей прибыли от реализуемой продукции).

4. Сортинг покупателей по категориям:

a) основные;

б) второстепенные;

в) высокоприбыльные;

г) низкоприбыльные.

К основнылм покупателям относятся субъекты, финансовые потоки которых приносят большую часть прибыли компании. В эту группу входят клиенты, на долю которых приходится больше n-го процента от общей прибыли. Для каждой компании этот барьер индивидуален, в зависимости от специфики организации и количества клиентов.

Bторостепенные - клиенты, приносящие незначительную часть прибыли с реализуемой продукции.

Высокоприбыльные - клиенты, приносящие доход с единицы продукции выше среднего значения. Среднее значение можно рассчитать по формуле

$$
\mathrm{M}=Ц-\mathrm{C},
$$

где $\mathrm{M}$ - средняя прибыль на единицу продукции,

Ц - средняя отпускная цена продукта,

$\mathrm{C}$ - себестоимость продукта.

Низкоприбыльные - покупатели, приносящие маржу на единицу продукции ниже среднего значения.

Рассмотрим каждый шаг более подробно на примере.

Компания «Ү» имеет 10 клиентов, формирующих 100 \% прибыли, в данном случае не учитываются непроизводственные доходы организации (кредитование, сдача в аренду и т. д.). Далее покупатели дифференцируются, в соответствии с приносимой прибылью. Для компании «Ү» это представлено в табл. 1. Затем проводится определения доли прибыли от конкретного поставщика и производится сортинг клиентов по группам:
а) основные;
б) второстепенные;
в) высокоприбыльные;
г) низкоприбыльные. 
Таблица 1

\begin{tabular}{|c|c|c|}
\hline № & Наименование клиента & Процент от прибыли, приходящийся на каждого клиента (\%) \\
\hline 1 & Кл1 & 29,5 \\
\hline 2 & Кл2 & 20,1 \\
\hline 3 & Кл3 & 18,2 \\
\hline 4 & Кл4 & 9,6 \\
\hline 5 & Кл5 & 8,1 \\
\hline 6 & Кл6 & 4,5 \\
\hline 7 & Кл7 & 3,8 \\
\hline 8 & Кл8 & 2.7 \\
\hline 9 & Кл9 & 2,2 \\
\hline 10 & Кл10 & 1,3 \\
\hline
\end{tabular}

После какого уровня прибыли следует относить к клиента к основнылм и второстепенныли, решает эксперт, исходя из того, что чем больше у компании клиентов и чем меньше разница между приносимой ими доходом, тем ниже уровень разности процента прибыли между основными и второстепенными поставщиками. В примере этот барьер имеет границу 5 \%.

Для наглядности группы сводятся в табл. 2.

Специфика анализа заключается в том, что крупные покупатели могут приносить высокий совокупный доход, но не являться высокодоходными на единицу продукции. Как видно из табл. 2, одни клиенты попадают в несколько колонок одновременно, таким образом клиенты Кл1, Кл2, Кл6 приносят прибыль ниже средней (с единицы продукции), хотя в то же время клиенты Кл1 и Кл2 входят в категорию основных по доходности. Покупатели Кл5, Кл7, Кл10 дают маржу выше среднего значения. Все остальные (Кл3, Кл4, Кл8, Кл9) - примерно равную среднюю доход- ность с единицы продукции.

II. Основной задачей второго этапа является определение степени влияния между организацией и её клиентами. Для этого необходимо произвести следующие операции:

1. Отбор покупателей.

2. Анализ формирования доли прибыли отдельного клиента.

3. Сбор информации и определение издержек клиента приходящихся на продукцию компании.

4. Определение баланса уровня влияния в отношениях между компанией и клиентом.

Первый шаг второго этапа заключается в фильтрации покупателей из сформированной выше табл. 2. Это обусловлено двумя причинами: фокусирование внимания на максимально доходных потребителях и снижение издержек анализа, исключая малозначимых агентов. Исходя из этого, при дальнейшем анализе объектом исследования будут

Таблица 2

\begin{tabular}{|c|c|c|c|c|}
\hline № & Основные & Второстепенные & Высокоприбыльные & Низкоприбыльные \\
\hline 1 & Кл1 & & & Кл1 \\
\hline 2 & Кл2 & & & Кл2 \\
\hline 3 & Кл3 & & & \\
\hline 4 & Кл4 & & Кл5 & Кл6 \\
\hline 5 & Кл5 & & & \\
\hline 6 & & Кл6 & Кл7 & \\
\hline 7 & & Кл7 & & \\
\hline 8 & & Кл8 & Кл10 & \\
\hline 9 & & Кл9 & Кл10 & \\
\hline 10 & & &
\end{tabular}


взаимоотношения между наиболее ценными клиентами и компанией. Клиентов, относящихся к второстеnенной группе, не обязательно подвергать анализу ${ }^{3}$, так как это сопровождается нерентабельными издержками. Следовательно, из примера с компанией «Ү» анализировать клиентов Кл6, Кл7, Кл8, Кл9, Кл10 нерационально.

Дальнейшие операции будут проводиться с основныли клиентами: Кл1, Кл2, Кл3, Кл4, Кл5.

Следующий шаг отчасти был проделан на первом этапе, при рассмотрении финансовых потоков, идущих в компанию. В данном случае необходимо установить долю прибыли от реализации продукта конкретному покупателю. В компании «Ү» потоки долей прибыли от клиентов формируются следующим образом:

$$
\begin{gathered}
\text { Кл1 }=29,5 \% ; \\
\text { Кл2=20,1\%; } \\
\text { Кл3=18,2\%; } \\
\text { Кл4=9,6\%; } \\
\text { Кл5 = 8,1 \%; } \\
\text { Итого: 85,5 \% }
\end{gathered}
$$

В итоге анализируются пять клиентов, генерирующих 85,5 \% прибыли.

Следующий шаг состоит из сбора информации относительно доли издержек клиента, приходящейся на продукцию компании. Основная задача заключается в определении объема затрат клиента на продукцию компании от величины его общих затрат. Необходимая информация может быть получена из открытых источников, так и предоставлена самим клиентом. После сбора и анализа сопутствующей информации можно с достаточной точностью сказать, какая доля от общих внешних издержек покупателя связана с продукцией компании-производителя.

В примере с компании «Ү» процент затрат клиентов на продукцию компании «Ү» следующий:

$$
\begin{aligned}
& \text { Кл1 }=10,0 \% ; \\
& \text { Кл2 }=20,0 \% ; \\
& \text { Кл3 }=40,0 \% ; \\
& \text { Кл4=10,0\%; }
\end{aligned}
$$

3 В анализ могут быть включены высокоприбыльные покупатели при условии, что это перспективные и растущие агенты и в будущем могут перейти в разряд основных.

$$
\text { Кл5 }=25,0 \% \text {. }
$$

На заключительном шаге II-го этапа производится сравнение процентных показателей, исходя из следующего принципа: чем выше доля прибыли компании от отдельного клиента, и чем ниже издержки последнего, приходящиеся на компанию, тем большей силой влияния обладает клиент и наоборот.

Процедура анализа взаимодействия между компанией и клиентом заключается в сравнении доли прибыли компании, приходящейся от реализации продукта соответствующему клиенту, и процент издержек последнего, приходящихся на продукцию компании.

В примере с компанией «Ү» в табл. 3 занесены показатели прибыли, поступающей от конкретного клиента, а также количество издержек покупателей, приходящихся на продукцию компании.

Сравнивая процентные значения, получаем следующие неравенства:

$$
\begin{aligned}
& \text { 1. } 29,5>10 \\
& \text { 2. } 20,1 \approx 20 \\
& \text { 3. } 18,2<40 \\
& \text { 4. } 9,6 \approx 10 ; \\
& \text { 5. } 8,1<25
\end{aligned}
$$

Итак, чем ниже доля прибыли компании, приходящаяся от клиента и выше издержки клиента на приобретаемый продукт, тем более сильную позииию занимает компания. Исходя из вышеприведенных неравенств, справедливо утверждать, что более выгодное положение у компании при взаимодействии с клиентами Кл3, Кл5. Клиент Кл1 обладает более выгодной позицией. Равенство во втором и четвертом неравенстве говорит о равноправии сторон, такие отношения в большей степени являются партнерскими.

Делая вывод, можно сказать, что, с одной стороны, каждая компания стремится обладать монопольной властью, с другой, при наличии равноправного партнера возникают трудности, толкающие компании к обоюдному развитию. Равноправное развитие совместно с поставщиками и потребителями помогает избежать застоя и отставания от фирм конкурентов. При равноправных отношениях клиенты выступают в роли оппонентов, что делает компанию более конкурентоспособной. Таким образом, в долгосрочной перспективе равноценные (на уровне 50/50) взаимоотношения со своими клиентами наиболее выгодны для компании и клиентов. 
Таблица 3

\begin{tabular}{|c|c|c|}
\hline $\begin{array}{c}\text { Наименование } \\
\text { Клиентов }\end{array}$ & $\begin{array}{c}\text { Прибыль компании «Ү» от } \\
\text { конкретного клиента (\%) }\end{array}$ & $\begin{array}{c}\text { Издержки потребителей приходящиеся на } \\
\text { продукцию компании «Ү» (\%) }\end{array}$ \\
\hline Кл1 & $29,5 \%$ & $10 \%$ \\
\hline Кл2 & $20,1 \%$ & $20 \%$ \\
\hline Кл3 & $18,2 \%$ & $40 \%$ \\
\hline Кл4 & $9,6 \%$ & $10 \%$ \\
\hline Кл5 & $8,1 \%$ & $25 \%$ \\
\hline
\end{tabular}

\section{Библиографический список}

1. Mechanisms of support of export-oriented small enterprises: the regional aspect [Text] / UV. Busarina, S. S. Morkovina, S. V. Budkova, E. A. Kolesnichenko // Asian Social Science. - 2014. - Vol. 10 - № 23. - P. 95-101.

2. Methodological approach to the identification of predictive models of socio-economic processes for investment and innovative development of enterprises [Text] / T. L. Bezrukova, S. S. Morkovina, B. A. Bezrukov, E. G. Popkova // World Applied Sciences Journal. - 2013. - Vol. 26 - № 1. - P. 20-27.

3. Ershova, I. Methodical approaches to assessment of intellectual rent as a factor of innovative economy development [Text] / I. Ershova, I. Androsova, S. Morkovina // 3rd International multidisciplinary scientific conference on social sciences and arts sgem 2016. - 2016. - P. 425-430.

4. State stimulation of development of small entrepreneurship in developing countries [Text] / O. N. Kusakina, N. V. Bannikova, S. S. Morkovina, T. N. Litvinova // European Research Studies Journal. - 2016. - Vol. 19 - № 2S. P. 276-284.

5. Макаров, Е. И. Интеллектуальная собственность как реализованная инновация [Текст] / Е. И. Макаров, С. Н. Дьяконова // Инженерный вестник Дона. - 2012. - № 2. - С. 329-334.

6. Макаров, Е. И. Особенности определения стоимости объектов интеллектуальной собственности [Текст] / Е. И. Макаров, С. Н. Дьяконова // Научный вестник Воронежского государственного-архитектурно-строительного университета. Серия: Экономика и предпринимательство. - 2007. - № 5. - С. 51.

7. Морковина, С. С. АВС-анализ как инструмент оперативного планирования основной деятельности организаций [Текст] / С. С. Морковина, С. В. Фурсова // Экономический анализ: теория и практика. - 2012. - № 38. C. 2-9.

8. Development of methodological approaches to the efficiency analysis of territorial-industry cluster formation in the forest sector [Text] / S. S. Morkovina, E. G. Popkova, M. S. Santalova, A. V. Konstantinov // Asian Social Science. - 2014. Vol. 10 - № 23. - P. 81-93.

9. Котлер, Ф. Маркетинг менеджмент [Текст] / Ф. Котлер ; пер. с англ. - 11-е изд. - СПб. : Питер, 2003 - 800 с.

10. Портер, М. Е. Конкурентная стратегия: методика анализа отраслей и конкурентов [Текст] / М. Е. Портер ; пер. с англ. - М. : Альпина Бизнес Букс, 2005 - 464 с.

11. Томпсон-мл., А. Стратегический менеджмент: Концепции и ситуации для анализа [Текст] / А. Томпсон-мл., III. А. Дж. Стрикленд ; пер. с англ. - 12-е изд. - М. : Вильямс, 2006. - 928 с.

12. Хасси, Д. Стратегия и планирование: путеводитель менеджера [Текст] / Д. Хасси. - СПб. : Питер, 2001. $-384 \mathrm{c}$.

\section{References}

1. Busarina U.V., Morkovina S.S., Budkova S.V., Kolesnichenko E.A. Mechanisms of support of export-oriented small enterprises: the regional aspect, 2014, Vol. 10, no. 23. pp. 95-101.

2. Bezrukova T.L., Morkovina S.S., Bezrukov B.A., Popkova E.G. Methodological approach to the identification of predictive models of socio-economic processes for investment and innovative development of enterprises World Applied Sciences Journal, 2013, Vol. 26, no. 1. pp. 20-27. 
3. Ershova I., Androsova I., Morkovina S. Methodical approaches to the assessment of intellectual rent as a factor of the innovative economy development. 3rd International multidisciplinary scientific conference on social sciences and Arts sgem 2016. Economics and tourism, 2016, pp. 425-430.

4. Kusakina O.N., Bannikova N. V., Morkovina S.S., Litvinova T.N. State stimulation of development of small entrepreneurship in developing countries. European Research Studies Journal, 2016, Vol. 19, no. 2S, pp. 276-284.

5. Makarov E.I., D'yakonova S.N. Intellektual'naya sobstvennost' kak realizovannaya innovatsiya [Intellectual property as a realized innovation] Inzhenernyy vestnik Dona [The engineer's messenger of the Don], Rostov-on-Don, 2012, Vol. 20, no. 2, pp. 329-334. (In Russian).

6. Makarov E. I., D'yakonova S.N. Osobennosti opredeleniya stoimosti ob"ektov intellektual'noy sobstvennosti [Peculiarities of determining the value of intellectual property objects] Nauchnyy vestnik Voronezhskogo gosudarstvennogoarkhitekturno-stroitel'nogo universiteta. Seriya: Ekonomika i predprinimatel'stvo [Scientific herald of the Voronezh State University of Architecture and Civil Engineering. Series: Economics and Entrepreneurship] Voronezh, 2007. no. 5, p.51 (In Russian).

7. Morkovina S.S., Fursova S.V. AVS-analiz kak instrument operativnogo planirovaniya osnovnoy deyatel'nosti organizatsiy [ABC-analysis as a tool for operational planning of the main activities of organizations] Ekonomicheskiy analiz: teoriya $i$ praktika [Economic Analysis: Theory and Practice], Moscow, 2012, no. 38, pp. 2-9. (In Russian).

8. Morkovina S.S., Popkova E.G., Santalova M.S., Konstantinov A.V. Development of methodological approaches to the efficiency analysis of territorial-industry cluster formation in the forest sector. Asian Social Science, 2014, Vol. 10, no. 23, pp. 81-93.

9. Kotler F. Marketing menedzhment [Marketing Management]. Saint Petersburg, Vol. 11, 2003, 800 p. (In Russian).

10. Porter E. Maykl Konkurentnaya strategiya: metodika analiza otrasley i konkurentov [Competitive strategy: a technique of the analysis of branches and competitors]. Moscow, 2005, 464 p. (In Russian).

11. Tompson-ml., Artur, A., Striklend III A., Dzh. Strategicheskiy menedzhment: Kontseptsii i situatsii dlya analiza [Strategic management: Concepts and situations for analysis]. Moscow, Vol. 12, 2006, 928 p. (In Russian).

12. Khassi D. Strategiya i planirovanie: putevoditel' menedzhera [Strategy and planning: manager's guide]. Saint Petersburg, 2001, 384 p. (In Russian).

\section{Сведения об авторах}

Макаров Евгений Иванович - заведующий кафедрой управления социально-экономическими системами и бизнес-процессами Воронежского филиала ФГБОУ ВО «РЭУ им. Г.В. Плеханова», доктор экономических наук, професcop, г. Воронеж, Российская Федерация; e-mail: ea_makarov@mail.ru.

Егоров Виктор Геннадьевич - аспирант кафедры управления социально-экономическими системами и бизнеспроцессами Воронежского филиала ФГБОУ ВО «РЭУ им. Г.В. Плеханова», магистр по направлению подготовки «Строительство», г. Воронеж, Российская Федерация; e-mail: Egorov.vrn@yandex.ru.

\section{Information about authors}

Makarov Evgeny Ivanovich - Head of department of management of social and economic systems and business processes of the Voronezh branch of Federal State Budget Educational Institution of Higher Education «Plekhanov Russian University of Economics», DSc in Economics, Professor, Voronezh, Russian Federation; e-mail: ea_makarov@mail.ru

Egorov Viktor Gennadievich. - post-graduate student of the Voronezh branch of Federal State Budget Educational Institution of Higher Education «Plekhanov Russian University of Economics», Master of the direction of preparation «Building», Voronezh, Russian Federation; e-mail: Egorov.vrn@yandex.ru 